\title{
Squamous Papillomatosis
}

National Cancer Institute

\section{Source}

National Cancer Institute. Squamous Papillomatosis. NCI Thesaurus. Code C9009.

A benign squamous neoplasm characterized by a papillary growth pattern, diffusely involving a specific anatomic site. 